\title{
To study the efficacy of sabina, cimicifuga and thalaspi bursa in management of cases of dysfunctional uterine bleedings
}

\author{
Parth Aphale*, Atul Rajgurav
}

\begin{abstract}
Department of Homoeopathic Pharmacy, Dr. D.Y. Patil Homoeopathic Medical College and Research Centre, Pune,
\end{abstract} Maharashtra, India

Received: 19 October 2016

Revised: 21 October 2016

Accepted: 15 November 2016

\author{
*Correspondence: \\ Dr. Parth Aphale, \\ E-mail: parth.aphale@gmail.com
}

Copyright: (c) the author(s), publisher and licensee Medip Academy. This is an open-access article distributed under the terms of the Creative Commons Attribution Non-Commercial License, which permits unrestricted non-commercial use, distribution, and reproduction in any medium, provided the original work is properly cited.

\begin{abstract}
Background: Abnormal uterine bleeding is one of the most common problems among pre and postmenopausal women. Virtually every woman will at some point in her lifetime experiences episodes of bleeding that are perceived as abnormal. Menstrual abnormalities are the cause of discomfort, inconvenience and disruption of healthy social and physical lifestyles among millions of women worldwide. Dysfunctional uterine bleeding is an abnormal bleeding from uterus in the absence of an organic pathology to account for. It commonly occurs in reproductive and perimenopausal age groups. When no systemic or local pelvic cause is evident to the clinician, histopathological examination of the endometrial biopsies remains the only alternative to reach the diagnosis. The following study intends to find out the incidence of various etiopathological factors in cases of dysfunctional uterine bleeding and to study the efficacy of Homoeopathic Medicines in cases of DUB.

Methods: 30 cases fitting the case definition were studied. Female patients above 35yrs. age showing abnormal bleeding from the uterus without any underlying pathology were selected for the study. Pregnant women, females below 35yrs., showing abnormal finding on USG were excluded from the study. Criteria for assessment were laid down and scoring was done and analyzed.

Results: Scoring was done before treatment and after treatment, $\mathrm{t}$ - test was applied. Null and alternative hypothesis were laid down. From the value of t-test, we reject the null hypothesis and accept the alternative hypothesis.

Conclusions: Homoeopathic medicines Sabina, Cimicifuga and Thalaspi Bursa were found effective in management of cases of DUB.
\end{abstract}

Keywords: Biopsies, DUB, Etiopathological factors, Endometrium, Menstrual disorder, Perimenopausal

\section{INTRODUCTION}

Dysfunctional uterine bleeding is abnormal uterine bleeding that, after examination and ultrasonography, cannot be attributed to the usual causes (structural gynecologic abnormalities, cancer, inflammation, systemic disorders, pregnancy, complications of pregnancy, use of oral contraceptives or certain drugs). Treatment is usually with hormone therapy, such as oral contraceptives, or with NSAIDs. ${ }^{1}$
Dysfunctional uterine bleeding (DUB), the most common cause of abnormal uterine bleeding, occurs most often in women $>45$ (> $50 \%$ of cases) and in adolescents (20\% of cases). About $90 \%$ of cases are anovulatory; $10 \%$ are ovulatory.

\section{Pathophysiology}

During an anovulatory cycle, the corpus luteum does not form. Thus, the normal cyclical secretion ofprogesterone does not occur, and estrogen stimulates the endometrium 
unopposed. Without progesterone, the endometrium continues to proliferate, eventually outgrowing its blood supply; it then sloughs incompletely and bleeds irregularly and sometimes profusely or for a long time. When this abnormal process occurs repeatedly, the endometrium can become hyperplastic, sometimes with atypical or cancerous cells.

In ovulatory DUB, progesterone secretion is prolonged; irregular shedding of the endometrium results, probably because estrogen levels remain low, near the threshold for bleeding (as occurs during menses). In obese women, ovulatory DUB can occur if estrogen levels are high, resulting in amenorrhea alternating with irregular or prolonged bleeding. ${ }^{2,3}$

\section{Complications}

Chronic bleeding may cause iron deficiency anemia.

If DUB is due to chronic anovulation, infertility may also be present.

\section{Etiology}

Anovulatory DUB can result from any disorder or condition that causes anovulation (see Table: Some Causes of Anovulatory Amenorrhea). Anovulation is most often

- Secondary to polycystic ovary syndrome

- Idiopathic (sometimes occurring when gonadotropin levels are normal).

Sometimes anovulation results from hypothyroidism.

During perimenopause, DUB may be an early sign of ovarian insufficiency or failure; follicles are still developing but, despite increasing levels of folliclestimulating hormone (FSH), do not produce enough estrogen to trigger ovulation. About $20 \%$ of women with endometriosis have anovulatory DUB due to unknown mechanisms.

\section{Ovulatory DUB may occur in}

- Polycystic ovary syndrome (because progesterone secretion is prolonged)

- Endometriosis, which does not affect ovulation.

Other causes are a short follicular phase and luteal phase dysfunction (due to inadequate progesterone stimulation of the endometrium); a rapid decrease in estrogen before ovulation can cause spotting. ${ }^{4-6}$

\section{Symptoms and Signs}

Compared with typical menses, bleeding may
- Occur more frequently (menses < 21 days apartpolymenorrhea)

- Involve more blood loss (> 7 days or $>80 \mathrm{~mL}$ ) during menses (menorrhagia, or hypermenorrhea)

- Occur frequently and irregularly between menses (metrorrhagia)

- Involve more blood loss during menses and frequent and irregular bleeding between menses (menometrorrhagia). ${ }^{7,8}$

\section{Diagnosis}

- Exclusion of other potential causes

- Hormone measurement [eg, thyroid-stimulating hormone (TSH), prolactin]

- Usually trans-vaginal ultrasonography and endometrial sampling

- Often sonohysterography and/or hysteroscopy.

Women should be evaluated for DUB when the amount or timing of vaginal bleeding is inconsistent with normal menses.

\section{Additional testing}

Trans-vaginal ultrasonography is done if women have any of the following

- $\quad$ Age $\geq 35$

- Risk factors for endometrial cancer (eg, obesity, diabetes, hypertension, polycystic ovary syndrome, chronic eugonadal anovulation, hirsutism, other conditions associated with prolonged unopposed estrogen exposure)

- Bleeding that continues despite use of empiric hormone therapy

- Pelvic organs that cannot be examined adequately during the physical examination

- Clinical evidence that suggests abnormalities in the ovaries or uterus.

These criteria include almost all women with dysfunctional uterine bleeding.

Trans-vaginal ultrasonography can detect structural abnormalities, including most polyps, fibroids, other masses, endometrial cancer, and any areas of focal thickening in the endometrium. If focal thickening is detected, further testing may be needed to identify smaller intrauterine masses (eg, small endometrial polyps, submucousmyomas). Sonohysterography (ultrasonography after saline is infused into the uterus) is useful in evaluating such abnormalities; it can be used to determine whether hysteroscopy, a more invasive test, is indicated and to plan resection of intrauterine masses. Or hysteroscopy may be done without sonohysteroscopy.

In endometrial sampling, only about $25 \%$ of the endometrium is analysed, but sensitivity for detecting 
abnormal cells is about 97\%. This test is usually recommended to rule out hyperplasia or cancer in women with any of the following:

- Age > 35 years with one or more risk factors for endometrial cancer (see above)

- Age < 35 years with multiple risk factors for endometrial cancer (see above)

- Bleeding that is persistent, irregular, or heavy

- Irregular cycles that suggest chronic anovulatory bleeding

- Endometrial thickness that is $>4 \mathrm{~mm}$, focal, or irregular, detected during transvaginalultrasongraphy

- Inconclusive ultrasonography findings. ${ }^{9-11}$

\section{Bleeding}

Nonhormonal treatments have fewer risks and adverse effects than hormone therapy and can be given intermittently, when bleeding occurs. They are used mainly for heavy regular bleeding (menorrhagia). Choices include.

- NSAIDs, which reduce bleeding by 25 to $35 \%$ and relieve dysmenorrhea by reducing prostaglandin levels

- Tranexamic acid, which inhibits plasminogen activator, reducing menstrual blood loss by 40 to $60 \%$.

Hormone therapy (eg, oral contraceptives, progestogens) is often tried first in perimenopausal women. This therapy does the following:

- Suppresses endometrial development

- Reestablishes predictable bleeding patterns

- Decreases menstrual flow.

Hormone therapy is usually given until bleeding has been controlled for a few months.

Oral contraceptives (OCs) are commonly given. OCs, used cyclically or continuously, can control dysfunctional bleeding. Limited data suggest that they do the following:

- Decrease menstrual blood loss by 40 to $50 \%$

- Decrease breast tenderness and dysmenorrhea

- Decrease risk of uterine and ovarian cancer.

Combination formulations consisting of an estrogen and a progestin or a progestin alone may be used. Risks of an OC depend on the type of OC and on patient factors.

Hysteroscopy with D and C may be therapeutic as well as diagnostic; it may be the treatment of choice when anovulatory bleeding is severe or when hormone therapy is ineffective. Structural causes such as polyps or fibroids may be identified or removed during hysteroscopy.
Hysterectomy, abdominal or vaginal, may be recommended for patients who decline hormone therapy or who, despite other treatments, have symptomatic anemia or poor quality of life caused by persistent, irregular bleeding.

\section{Key points}

- Anovulatory DUB is the most common cause of abnormal uterine bleeding

- Test for treatable causes of bleeding; tests may include a pregnancy test, $\mathrm{CBC}$, measurement of hormone levels (TSH, prolactin, progesterone), and often ultrasonography and endometrial sampling

- In women at risk, check for and treat endometrial hyperplasia

- If drugs are needed to control bleeding, prescribe NSAIDs, tranexamic acid, OCs, or other hormones, which are usually effective. ${ }^{12,13}$

The objective of this study was to study the efficacy of homoeopathy in cases of DUB.

- To study in detail the efficacy of homoeopathy in managing DUB

- To cut short the total time duration required for complete eradication of the symptoms and achieving cure

- To prevent any acute complications with the help of homoeopathic intervention

- To prevent recurrence of the acute attack with the help of homoeopathic medicines.

\section{Purpose of selection}

Abnormal uterine bleeding is one of the most common problems among pre and postmenopausal women. Virtually every woman will at some point in her lifetime experiences episodes of bleeding that are perceived as abnormal.

A menstrual abnormality was the cause of discomfort, inconvenience and disruption of healthy social and physical lifestyles among millions of women worldwide.

It can be caused by a wide variety of disorders, some of which represent normal physiological process, while others are a sign of serious underlying pathology.

Managing DUB is a very challenging job. They have a sudden onset and short or prolonged duration. There is no time to wait and watch. In acute cases, both the remedy and the potency have to hit the bull's eye. In case of chronic cases, he/she can buy some time in case the prescription does not hit right in the first go, it may be in terms of remedy as well as potency. But it is not the case in acute cases. Acute DUB is even more difficult to treat since there is added pressure to make the patient fine within a very short span of time. The patient is in acute 
distress and wants instant relief of the symptoms. Acute DUB hampers all the day-to-day activities.

If not completely treated and cured, it may lead to complications which we want to avoid.

\section{METHODS}

Data obtain from the OPD's of the authors. Type of study was 30 cases were being studied over a period of 6 months. The subject will be studied through various books on homoeopathic posology, selection of potency and high potencies, journals, that the subject is thoroughly known.

Clinical study, OPD patient's data will be collected. Each patient's data will be processed in standardized format with the following steps-

Data receiving, each patient will be given adequate time and data will be elicited in a comprehensive manner as to elicit proper patient's picture in the disease.

Processing of the case will be done as per the principles and guidelines of homoeopathy.

References from homoeopathic material medica, repertory, will be availed for selection of remedy. All cases will be followed up for sufficient period required as per the guidelines from Organon and Homoeopathic Philosophy.

References from Repertory and Materiamedica will be availed for selection of single remedy out of the indicated group of remedies.

\section{All cases of DUB will be taken in to consideration.}

Dysfunctional uterine bleeding is abnormal uterine bleeding that, after examination and ultrasonography, cannot be attributed to the usual causes (structural gynecologic abnormalities, cancer, inflammation, systemic disorders, pregnancy, complications of pregnancy, use of oral contraceptives or certain drugs).

Pilot study was design all acute cases which satisfy the case definition will be studied.

\section{Inclusive criteria}

- Age > 35 years with one or more risk factors for endometrial cancer

- Bleeding that is persistent, irregular, or heavy

- Irregular cycles that suggest chronic anovulatory bleeding

- Endometrial thickness that is > $4 \mathrm{~mm}$, focal, or irregular, detected during trans-vaginal ultrasongraphy

- Inconclusive ultrasonography findings
- Risk factors for endometrial cancer (eg, obesity, diabetes, hypertension, polycystic ovary syndrome, chronic eugonadal anovulation, hirsutism, other conditions associated with prolonged unopposed estrogen exposure)

- Bleeding that continues despite use of empiric hormone therapy

- Pelvic organs that cannot be examined adequately during the physical examination

- Clinical evidence that suggests abnormalities in the ovaries or uterus

- These criteria include almost all women with dysfunctional uterine bleeding.

\section{Exclusion criterias}

- Pregnant women

- Women on hormonal preparations.

- Women with organic changes in the uterus like fibroids, $\mathrm{Ca}$

- Menopause

- Females $<35$ years.

\section{Criteria for assessment will be}

- Complete disappearance of symptoms

- Patient in general

- No new symptoms.

\section{Cure}

Complete disappearances of symptom-complex within 72 hours.

\section{Good response}

- Improvement more than $70 \%$ of the symptom complex within 72 hours.

- Incomplete regression of the pathology, if any.

\section{No relief/worse}

- No improvement whatsoever within 2-3 days.

For ease of evaluation I have graded the follow up thus,

- Grade I - Complete removal of symptoms and signs within definitive period of each case

- Grade II - Complete removal of symptoms but signs remain

- Grade III - Some symptoms and signs remain

- Grade IV - No relief, followed by natural recovery or progressive worsening of case.

\section{Selection and administration of drugs}

Selection of remedy will be done after verification from standard text books of Materiamedica. Dose and 
repetition will be based on principles of Homoeopathic posology. High potency will be selected. Route of administration will be oral. All cases will be given placebo once improvement is seen (aphorism 246).

\section{Case recording}

All the cases will be recorded as per the standard case performa as prescribed by the institute. Unnecessary details of irrelevant chronic phenomenon were not taken as per the guidelines given in aphorism 92 .

\section{Statistical analysis}

Effectiveness of homoeopathic medicines in migraine was assessed according to statistical principles on the basis of change in score taken before and after treatment with homoeopathy. The data obtained from patients before introduction of variable has formed the control which was compared with outcome of the symptom complex through the objective assessment after homoeopathic treatment in same patients. Since efficacy of Homoeopathic medicines on patients having definite group of signs and symptoms before administration of homoeopathic remedy were taken as control and compared with their symptomatology after administration of similar remedy as response, no separate strategies of elimination of error or bias like use of controls, randomization, cross over design or placebo group and blinding techniques were used in this study.

The following marks were given to the clinical features of cases.

Table 1: Scores before treatment.

\begin{tabular}{|ll|}
\hline Clinical features & Score \\
\hline Presence of sign & 3 \\
\hline Presence of symptom & 2 \\
\hline Presence of pathology & 4 \\
\hline
\end{tabular}

Table 2: Scores after treatment.

\begin{tabular}{|ll|}
\hline Clinical Features & Score \\
\hline Disappearance of sign & 2 \\
\hline Ameliorationof symptoms & 1 \\
\hline Aggravation of symptoms & 3 \\
\hline Disappearance of pathology & 4 \\
\hline
\end{tabular}

\section{RESULTS}

Table 3: Age distribution.

\begin{tabular}{|lll|}
\hline Age group & No. of patient & Percentage \\
\hline $35-40$ & 06 & 20 \\
\hline $41-45$ & 20 & 66.66 \\
\hline $46-50$ & 04 & 13.33 \\
\hline Total & $\mathbf{3 0}$ & $\mathbf{1 0 0 \%}$ \\
\hline
\end{tabular}

Age: the reported age group is also varied. The youngest patient is 38 years old and the oldest is 50 years old.

\section{Acute remedy used in $D U B$}

Table 4: Remedy action percentage.

\begin{tabular}{|ll|}
\hline Acute & Improvement \\
\hline Sabina & $90 \%$ \\
\hline Thalaspi Bursa & $84 \%$ \\
\hline Cimicifuga & $80 \%$ \\
\hline
\end{tabular}

\section{Statistics}

Statistical working

$\mathrm{X}=$ score before treatment $\mathrm{Y}=$ score after treatment

$\mathrm{A}=$ difference between the scores

$\overline{\mathrm{A}}=$ mean of the difference between the scores

$\mathrm{S}=\mathrm{S}$. D. of differences $\mathrm{S} \mathrm{E}=$ standard error of mean.

Now we assume

Ho- null hypothesis states that homoeopathy are not useful inDUB

H1 - alternate hypothesis states that homoeopathy are useful inDUB.

$\overline{\mathrm{A}}=\sigma \mathrm{a} / \mathrm{n}=5.66$

$S=\sqrt{ } \sigma(a-\bar{a})^{2} / n-1=\sqrt{ } 348.5 / 29=3.465$

$\mathrm{Se}=\mathrm{s} / \sqrt{ } \mathrm{n}=3.465 / 5.47=0.6334$

$\mathrm{T}=\overline{\mathrm{a}} / \mathrm{se}=5.66 / 0.6334=8.98$

Thus $\mathrm{t}=8.98$

At $5 \%$ level for 29 degrees of level of freedom the value of $\mathrm{t}=2.05$

At $1 \%$ level for 29 degrees of level of freedom the value of $\mathrm{t}=2.76$.

Thus the value obtained is more than the above values so; we reject the null hypothesis and accept the alternative hypothesis.

Therefore homoeopathy is useful in dub. 
Table 5: Distribution of scores before and after Homoeopathic treatment.

\begin{tabular}{|llllll|}
\hline Case No. & $\mathbf{X}$ & $\mathbf{Y}$ & $\mathbf{X}-\mathbf{Y}$ & $\mathbf{A}-\mathbf{A}$ & $(\mathbf{A}-\overline{\mathbf{A}})^{2}$ \\
\hline 1 & 19 & 11 & 8 & 2.34 & 5.47 \\
\hline 2 & 6 & 3 & 3 & -2.66 & 7.07 \\
\hline 3 & 14 & 9 & 5 & -0.66 & 0.43 \\
\hline 4 & 9 & 7 & 2 & -3.66 & 13.39 \\
\hline 5 & 4 & 3 & 1 & -4.66 & 21.71 \\
\hline 6 & 10 & 2 & 8 & 2.34 & 5.47 \\
\hline 7 & 17 & 8 & 9 & 3.34 & 11.15 \\
\hline 8 & 8 & 6 & 2 & -3.66 & 13.39 \\
\hline 9 & 15 & 12 & 3 & -2.66 & 7.07 \\
\hline 10 & 12 & 5 & 7 & 1.34 & 1.79 \\
\hline 11 & 15 & 6 & 9 & 3.34 & 11.15 \\
\hline 12 & 23 & 12 & 11 & 5.34 & 28.51 \\
\hline 13 & 12 & 5 & 7 & 1.34 & 1.79 \\
\hline 14 & 13 & 10 & 3 & -2.66 & 7.07 \\
\hline 15 & 14 & 13 & 1 & -4.66 & 21.71 \\
\hline 16 & 10 & 9 & 1 & -4.66 & 21.71 \\
\hline 17 & 12 & 4 & 8 & 2.34 & 5.47 \\
\hline 18 & 16 & 12 & 4 & -1.66 & 2.75 \\
\hline 19 & 15 & 9 & 6 & 0.34 & 0.11 \\
\hline 20 & 18 & 10 & 8 & 2.34 & 5.47 \\
\hline 21 & 12 & 8 & 4 & -1.66 & 2.75 \\
\hline 22 & 12 & 6 & 6 & 0.34 & 0.11 \\
\hline 23 & 12 & 2 & 10 & 4.34 & 18.83 \\
\hline 24 & 13 & 2 & 11 & 5.34 & 28.51 \\
\hline 25 & 18 & 11 & 7 & 1.34 & 1.79 \\
\hline 26 & 14 & 5 & 9 & 3.34 & 11.15 \\
\hline 27 & 15 & 9 & 6 & 0.34 & 0.11 \\
\hline 28 & 10 & 10 & 0 & -5.66 & 32.03 \\
\hline 29 & 13 & 13 & -5.66 & 28.51 \\
\hline 30 & 17 & 6 & 5.34 & \\
\hline & & & & & \\
\hline
\end{tabular}

\section{DISCUSSION}

All 30 cases were selected satisfying the case definition. Maximum numbers of cases were found to be between the age group 40-45 years. Number of patients above 45 years was comparatively less may be due to beginning of menopause.

Investigations like USG were carried out to rule out any underlying pathology like uterine fibroids. Depending upon the type of flow, presence of clots, and pain Sabina, Thalaspi Bursa and Cimicifuga were prescribed to the respective patients strictly according to Homoeopathic principles.

It was observed that Sabina was more useful wherein the flow was bright red in colour, profuse, prolonged with pain radiating from back to the front of the abdomen, flow on least exertion. Thalaspi bursa was observed to work well where the flow was dark in colour, gushing, with clots++, one period stopped and the other started with very little or no gap in between. ${ }^{14-16}$

Cimicifuga was seen to be useful in cases of DUB with dysmenorrhea wherein the pain radiated from the back down the thighs, bleeding profuse dark coagulated. ${ }^{14-16}$

\section{Boricke's Matria medica states}

\section{Sabina}

Female complaints - menses profuse, bright. - Uterine pains extend into thighs. - Threatened miscarriage. Sexual desire increased. - Leucorrhoea after menses, corrosive, offensive. - Discharge of blood between periods, with sexual excitement. (Ambr) - Retained placenta; intense after-pains. - Menorrhagia in women who aborted readily. - Inflammation of ovaries and uterus after abortion. - Promotes expulsion of moles from uterus. (Canth) - Pain from sacrum to pubis, and from below upwards shooting up the vagina. - Haemorrhage; 
partly clotted; worse from least motion. - Atony of uterus. $^{14,16}$

\section{Thalaspi bursa}

Female complaints: metrorrhagia; too frequent and copious menses. - Haemorrhage, with violent uterine colic. - Every alternate period very profuse. Leucorrhoea before and after menses; bloody, dark, offensive; stains indelibly. Sore pain in womb on rising. Scarcely recovers from one period before another begins. $^{14,16}$

\section{Cimicifuga}

Female complaints: pain immediately before menses. Menses profuse, dark, coagulated, offensive with backache, nervousness; always irregular. - Ovarian neuralgia. - Pain across pelvis, from hip to hip. - Afterpains, with great sensitiveness and intolerance to pain. Infra-mammary pains worse, left side. ${ }^{14,16}$

None out of the total 30 cases showed any adverse effects.

It was observed that at the end of the study, their menses had become more regular, less painful, duration was shortened and they could carry out their regular chores just like before even during menses.

\section{CONCLUSION}

Out of the 30 cases which were evaluated, 23 cases showed improvement within 72 hours while 7 cases showed good response (more than $70 \%$ improvement within 72 hours). In none of the 30 cases, was there any acute complication. Total time duration required for complete eradication of symptoms was also greatly reduced with the help of homoeopathic medicines. Statistical analysis also shows that there is great difference in scores before treatment and after treatment.

Therefore, according to the 30 cases I have studied, I can say that homoeopathic medicines are very much useful in treatment of DUB. This inference is not only for statistical purpose but it gives us guidelines for prescribing in DUB. This will then be a feather in cap for Homoeopathy.

This will also make people get over the myth that Homoeopathy takes very long to act and has little or no role in treating emergency like DUB. It should be always kept in mind that the above mentioned 3 study drugs are just to test their efficacy with respect to their clinical Materia Medica. But these are not the whole and sole drugs for DUB. These are the forerunners in such cases which will help and guide the new comers in Homoeopathic Practice to treat cases of DUB. Thorough case taking, building up the totality and prescribing on the basis of laws of similar is and will always be the golden rule for prescribing.

\section{ACKNOWLEDGEMENTS}

Authors would like to thanks respected Dr. D.B. Sharma, Principal, Dr. DYPHMCRC, Pune for giving us this opportunity to take up this research project and test the efficacy of homoeopathy in DUB. We also would like to thank the ethics committee of our college for accepting this research project.

\section{Funding: No funding sources}

Conflict of interest: None declared

Ethical approval: The study was approved by the Institutional Ethics Committee

\section{REFERENCES}

1. Fluhmann CF. Textbook of Menstrual Disorders and Treatment; 1956:1-4.

2. William BJ. Robertson. The Abnormal Menstrual Cycle, Textbook of Endometrium, Butterworth; 1981:45-72.

3. Schroeder R. Endometrial hyperplasia in relation to genital function. Amer J Obstetr Gynaecol. 1954;68(1):294-309.

4. DasAnusuya, Chugh S. Dysfunctional uterine bleeding; a clinicopathological study. J Obstetr Gynaecol India. 1964;14(2):343-7.

5. Traut HF, Kuder. Irregular shedding and irregular ripening of the endometrium. Surg Gynaecol Obstetr. 1935;61(2):145-54.

6. Gusberg SB, Kaplan AL. Precursors of corpus cancer-4, adenomatous hyperplasia as stage 0 carcinoma of endometrium. Amer J Obstetr Gynaecol. 1963;87(5):662-76.

7. Noyes RW, Hertig AT, Rock J. Dating the endometrial biopsy. Fertil Steril. 1950;1:3.

8. Devi PK, Sutaria UD. Functional uterine bleeding. J Obstetr Gynaecol India. 1964;14(2):347-51.

9. Charusheela, Doifode, Fernandes K. Study of thyroid dysfunction in patients with dysfunctional uterine bleeding. J Obstetr Gynaecol India. 2001;51(2):93-5.

10. Vilos GA, Lefebvre G, Graves GR. Guidelines for management of abnormal uterine bleeding, SOGC, Clinical Practice Guidelines. J Obstetr Gynaecol Canada. 2001;146:1-4.

11. Revel A, Shushan A. Hysteroscopy with endometrial biopsy is the gold standard investigation for abnormal uterine bleeding. Human Reproduct. 2002;17(8):1947-9.

12. Dhangal G. A study of endometrium of patients with abnormal uterine bleeding at Chitwan valley, Katmandu University Med J. 2003;1(2):110-2.

13. Albers JR, Hull SK, Wesley RM. Abnormal uterine bleeding, 2008. Available at downloaded from www.aafp.org/afp. Assessed on 24 September 2009. 
14. Boericke W. Homoeopathic Materia Medica and Repertory. B Jain Publishers New Delhi. Edition-9, 1999, p-140, 780, 820.

15. Hughes R. The Principles and practice of homoeopathy, 4th ed, Ch XXVIII, B. Jain Pub (P) Ltd, 2000, p - 400-401.
16. Kent JT. Repertory of Homoeopathic Materia Medica; B. Jain Publishers New Delhi. 9th Edition, 2000, p-980, 1230, 1146.

Cite this article as: Agrawal S, Das V, Agarwal A, Pandey A. Fetal Doppler for prediction of adverse perinatal outcome in preeclampsia in a low resource setting. Int J Reprod Contracept Obstet Gynecol 2017;6:133-40 